\title{
BMJ Open Innovative measurement of routine physiological variables (heart rate, respiratory rate and oxygen saturation) using a remote photoplethysmography imaging system: a prospective comparative trial protocol
}

\author{
Edem Allado (1) ,1,2,3 Mathias Poussel (D) ,1,2 Anthony Moussu, ${ }^{1,3}$ \\ Véronique Saunier, ${ }^{4}$ Yohann Bernard, ${ }^{4}$ Eliane Albuisson, ${ }^{3,4,5}$ Bruno Chenuel ${ }^{1,2}$
}

To cite: Allado E, Poussel M, Moussu A, et al. Innovative measurement of routine physiological variables (heart rate, respiratory rate and oxygen saturation) using a remote photoplethysmography imaging system: a prospective comparative trial protocol. BMJ Open 2021;11:e047896. doi:10.1136/ bmjopen-2020-047896

- Prepublication history for this paper is available online. To view these files, please visit the journal online (http://dx.doi. org/10.1136/bmjopen-2020047896).

Received 11 December 2020 Accepted 04 August 2021

Check for updates

(c) Author(s) (or their employer(s)) 2021. Re-use permitted under CC BY-NC. No commercial re-use. See rights and permissions. Published by BMJ.

For numbered affiliations see end of article.

Correspondence to

Dr Edem Allado;

e.allado@chru-nancy.fr

\section{ABSTRACT}

Introduction Physiological signals are essential for assessing human health. The absence of a medical device to carry out these measurements remotely is one of the main limitations of telemedicine. Remote photoplethysmography imaging (rPPG) makes it possible to use a camera video to measure some of the most valuable physiological variables: heart rate $(\mathrm{HR})$, respiratory rate (RR) and oxygen saturation $\left(\mathrm{SpO}_{2}\right)$. Our objective was to evaluate the value of such remote measurements compared with existing contact point measurements techniques in real-life clinical settings.

Methods and analysis Prospective hospital-based study that will recruit 1045 patients who require a pulmonary function test. For each patient, measurements of $\mathrm{HR}, \mathrm{RR}$ and $\mathrm{SpO}_{2}$, using a standard acquisition system, will be carried out concomitantly with the measurements made by the rPPG system. 30,60 and 120 s time frames will be used to take measurements. Age, gender and skin phototype will also be collected. The intraclass coefficient correlation will be performed to determine the accuracy and precision of the rPPG algorithm readings.

Ethics and dissemination The study protocol has been approved by the French Agency for the Safety of Health Products (ANSM registration no. ID RCB 2020-A0242831 ) and by a French ethics committee (CPP OUEST I-TOURS-2020T1-30 DM at 30 October 2020). Results will be published in peer-reviewed journals, at scientific conferences and through press releases.

Trial registration number NCT04660318.

\section{INTRODUCTION}

Monitoring physiological variables, such as heart rate (HR), respiratory rate (RR) and oxygen saturation $\left(\mathrm{SpO}_{2}\right)$, are crucial for assessing human health. Technological improvements in engineering have enabled the growth of modern bioinstrumentation technology solutions that have made noninvasive clinical physiological monitoring
Strengths and limitations of this study

- The study assesses a remote photoplethysmography imaging system in clinical practice.

- Inclusion of a large population (more than 1000 patients) of different ages, gender and skin phototype requiring outpatient care.

- The use of a metrology system (standard acquisition system) as a control measure.

- A limitation of the remote photoplethysmography imaging system is the need to have a quiet position for patient.

systems available at relatively low cost. ${ }^{1}$ Currently, commonly used systems are based on electrodes in direct contact with the subject's skin and are themselves connected to an acquisition station for signal collection and analysis. ${ }^{23}$

Advances in medicine have introduced a need to perform analyses as close as possible to the patient's environment. Indeed, conventional methods are poorly adapted to outpatient monitoring. Although wireless methods using electrodes exist, they are sources of a range of artefacts and are sometimes uncomfortable, especially when exercise needs to be assessed, making them barely applicable in current and real-life practices. ${ }^{4}$ In addition, the installation and removal of these systems constrain (or force) the patient to travel from home, which can be perceived as time-consuming.

Introduced in 1930, photoplethysmography techniques raised the possibility of measuring SpO2 and HR using the absorption of light by the blood to define these signals. ${ }^{5}$ In recent years, a new approach to 
Table 1 Inclusion and exclusion criteria

\begin{tabular}{ll}
\hline Inclusion criteria & Exclusion criteria \\
\hline $\begin{array}{l}\text { Patients requiring } \\
\text { pulmonary function testing }\end{array}$ & Patients with stable clinical \\
Age $>18$ years & $\begin{array}{l}\text { Pregnant women or women of } \\
\text { childbearing potential without } \\
\text { effective contraception }\end{array}$ \\
\hline
\end{tabular}

photoplethysmography to measure physiological variables without contact has been developed. ${ }^{6}$ This technique, called remote photoplethysmography imaging (rPPG), uses the different red-green-blue colour spectra of facial skin tissue captured by a camera video to determine a plethysmography signal. ${ }^{7-13}$ Verkruysse et al also highlighted the feasibility of using a natural or artificial light source to perform measurements via PPPG. $^{7}$ First analysed to evaluate $\mathrm{HR}$ with an excellent correlation with intraclass correlation coefficient (ICG) of 0.90 , they have more recently also been able to identify RR. ${ }^{12} 14$

Although these systems have been technically validated, they have not been clinically evaluated in routine health settings. This clinical trial aims to assess parameter measurements by rPPG compared with a standard acquisition system in real-life conditions.

\section{METHODS}

\section{Design and study population}

This is an interventional, prospective, hospital-based study from a French hospital (University Hospital of Nancy). We will include 1045 adult patients managed in the Respiratory Function Exploration and Sports Medicine Department who require a pulmonary function test.
The patient selection criteria were defined to include stable outpatients and inpatients. Patients in intensive care or generally unstable are excluded Main inclusion and exclusion criteria are summarised in table 1.

\section{Protocol: research progress}

The study is to be initiated from January 2021 with an anticipated end for subject recruitment in July 2022. Study completion is scheduled for December 2022

Following validation of the inclusion criteria, all selected patients will be informed by investigator about the study and will provide written consent to participate (table 1). Patients underwent a specific physical examination to collect gender, age, body mass index, Fitzpatrick phototype, patient history, resting HR, RR. ${ }^{15}$

Following the pulmonary lung function assessment and a period of more than $10 \mathrm{~min}$ of rest, patients will be equipped with standard acquisition systems. Then they will be seated on a chair, at rest in a comfortable and quiet position, in front of a computer using a webcam and rPPG system. HR, RR and $\mathrm{SpO}_{2}$ measurements using the rPPG system and the standard acquisition system will be performed on the same time basis. (figure 1). Readings and recording will be realised for different time frames: 30, 60 and $120 \mathrm{~s}$. The duration of the patient's participation in the study is estimated at 15 min for all recordings.

\section{Medical device}

All the capture systems are synchronised from a single monitor to allow an assessment of the concordance of physiological variables across a common time base. The reference device is a set of approved and validated sensors to feed an analog-to-digital converter (PowerLab Acquisition Station, ADInstruments, Dunedin, New Zealand).

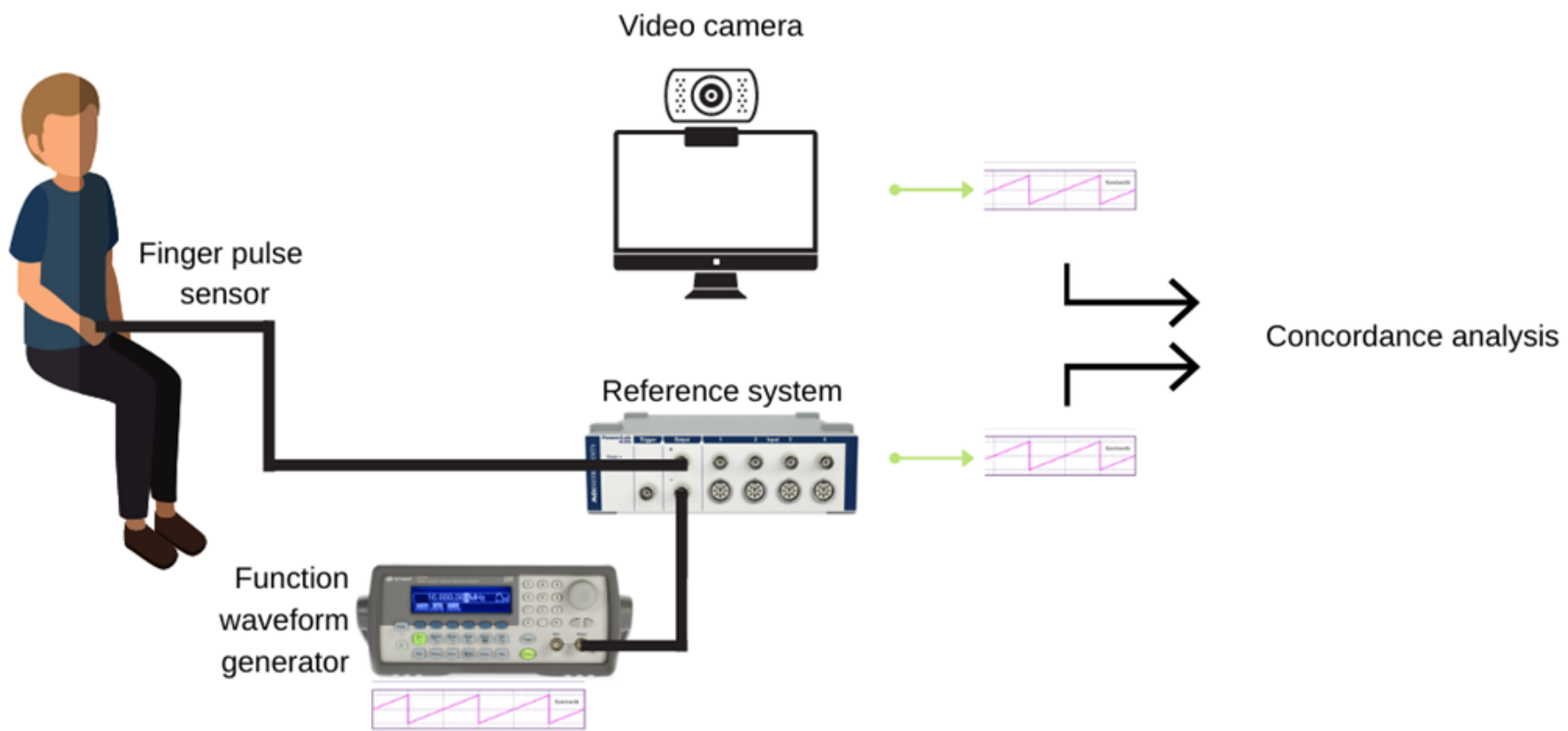

Figure 1 Parameter measurements procedure using a standard acquisition system and the remote photoplethysmography imaging system. 
This last acquisition chain enables synchronisation of all the signals on the same time base.

- HR will be obtained from an ECG system (unit: heart beats per minute (bpm))

- RR will be obtained from a transducer. This transducer contains a flexible sensing element bonded to the inside of a fabric belt (unit: respirations per minute (rpm))

- $\mathrm{SpO}_{2}$ will be obtained from an oximeter (oxygensaturated haemoglobin relative to total haemoglobin (unsaturated and saturated) in the peripheral blood-unit: per cent (\%)).

- Caducy Medical Device Software is the evaluated measurement system using rPPG. This software is based on an algorithm developed by the company I-Virtual. To make a measurement, the algorithm is based on three successive steps $^{16}$ :

Phase 1: Region of interest (ROI) determination: analysis area (head/face).$^{78}$ The ROI is the area of skin on the face that will allow analysis.

Phase 2: Each image from the ROI will benefit from a measurement of the red, green and blue colour spectra.

Phase 3: Measurement of the fluctuation of these spectra during a defined period with the intervention of an algorithm to define the physiological parameters (HR, RR and SpO2).

\section{Primary endpoint}

- HR concordance analysis by a standard acquisition system (photoplethysmography of the finger) compared with rPPG with a recording time of $60 \mathrm{~s}$.

\section{Secondary endpoints}

- HR concordance analysis by ECG system compared with rPPG with a recording time of $120 \mathrm{~s}$.

- $\mathrm{SpO}_{2}$ concordance analysis by standard acquisition system (oximeter) compared with rPPG with a recording time of $120 \mathrm{~s}$.

- RR concordance analysis by standard acquisition system (gold standard) compared with rPPG with a recording time of $120 \mathrm{~s}$

\section{Statistical analysis}

To determine the HR, RR and $\mathrm{SpO}_{2}$, descriptive analyses will be conducted according to the nature and the distribution of the variables. Qualitative variables will be described with frequencies and percentages; quantitative variables will be reported as mean $\pm \mathrm{SD}$. The subclass correlation coefficient with the $95 \%$ CI will be used to measure the concordance between the two measuring systems. The ICC with a $95 \%$ CI will be used to measure the concordance between the two measuring systems. The analysis will be adjusted according to age, sex and the six skin phototypes. ${ }^{15}$ Analyses were performed using IBM SPSS Statistics V.23, and $p$ values $<0.05$ were considered statistically significant.

\section{Sample size}

With a given ICC of $\rho=0.85$ between the two groups of measures with a $95 \%$ CI of 0.05 width (two sided), the adjustment according to age, sex and skin phototype, and considering $10 \%$ of bad quality signals, the workforce is 1045 subjects. This number and the subjects recruited will allow a sufficiently wide distribution of values (in terms of different observed values and variety of subjects) for a good distribution of the results on the axes and thus a good estimation of the ICC.

\section{Data management}

Before the launch of this study, all investigators and members of the investigative team took part in Good Clinical Practice (GCP) workshops. The sponsor's clinical research associates from the University Hospital of Nancy (France) will perform periodic monitoring throughout the trial. The data required to meet the research objectives will be collected in case report forms (CRF) specifically designed for this study and will come from the patient's medical records and the results of the analysis of biological samples.

A database will be created specifically for this study and will use the double entry method. To ensure data quality and data concordance, two data entry technicians will independently feed data from the CRFs into the database. If inconsistencies are detected, a third investigator will decide on the data to be entered. The data manager will send queries to the investigative teams to correct or confirm outliers. Before any decision to freeze the database and to ensure a clean database, all CRF data will be incorporated into the database and all queries will be resolved. The final milestone will be the database lock.

\section{DISCUSSION}

The clinical trial results will determine $\mathrm{rPPG}$ precision and reliability in measuring the HR (primary endpoint) and obtain parameters to determine the validity of $R R$ and $\mathrm{SpO}_{2}$. To our knowledge, this is the first study to evaluate the rPPG system in a large sample of subjects. This will assess the limits of this new method of physiological measurement. Finally, we will be able to evaluate this innovative physiological measurement system in current practice, opening up new possibilities for telemedicine.

By using a simple computer webcam or a smartphone camera, it will be possible to provide additional information for teleconsultations to assess the patient's current baseline clinical condition. This valid physiological evaluation is particularly crucial in cardiorespiratory disease so as to make appropriate medical decisions. ${ }^{78}$ During the COVID-19 pandemic, telemedicine has been a major component of healthcare strategies, limiting SARS-CoV-2 exposure and providing needed care delivery to high-risk patients. ${ }^{1718}$ Then, the development of remote assessments, independent from instruments that the patient has to manage himself, metrologically validated and useful in real-life settings will play a key role to allow 
accurate telediagnosis or telemonitoring. This study will provide valuable insights in order to clarify the accuracy of measurements of physiological variables (HR, RR and SpO2) using an rPPG system in real-life clinical settings at rest, but further studies will be needed to evaluate their interest in real-life home-based conditions.

\section{Ethics and dissemination}

This study has received approval from the French Ethics Committee (CPP TOURS-Région Centre-Ouest 1-2020 T1-30 DM at 27 October 2020) and from the French Agency for the Safety of Health Products (ANSM registration no. I-RCB 2020-A02428-31).

It will be conducted according to the European GCP recommendations, the general ethical principles of the Declaration of Helsinki and specific French regulations.

Before inclusion, each patient will receive, both verbally and in writing, a full brief on the study objectives, its progress and its constraints, before giving their written consent to participate in the trial.

\section{Author affiliations}

${ }^{1}$ CHRU-Nancy, University Center of Sports Medicine and Adapted Physical Activity, Centre hospitalier régional universitaire de Nancy, Nancy, Lorraine, France ${ }^{2}$ Université de Lorraine, DevAH, Université de Lorraine, Nancy, Lorraine, France ${ }^{3}$ OMEOS, Nancy, Lorraine, France

${ }^{4}$ CHRU-Nancy, Direction de la Recherche Clinique et de I'Innovation, Centre hospitalier régional universitaire de Nancy, Nancy, Lorraine, France

${ }^{5}$ Université de Lorraine, CNRS, IECL, Université de Lorraine, Nancy, Lorraine, France

Contributors EA had the original idea. EAll and EAlb designed and conceived the protocol. BC, MP and EAll drafted the manuscript. BC, MP, AM, VS, YB and EAlb critically revised the manuscript for methodology and intellectual content. All authors approved the final version of this manuscript.

Funding The authors have not declared a specific grant for this research from any funding agency in the public, commercial or not-for-profit sectors.

Competing interests None declared.

Patient and public involvement Patients and/or the public were not involved in the design, or conduct, or reporting, or dissemination plans of this research.

Patient consent for publication Not required.

Provenance and peer review Not commissioned; externally peer reviewed.

Open access This is an open access article distributed in accordance with the Creative Commons Attribution Non Commercial (CC BY-NC 4.0) license, which permits others to distribute, remix, adapt, build upon this work non-commercially, and license their derivative works on different terms, provided the original work is properly cited, appropriate credit is given, any changes made indicated, and the use is non-commercial. See: http://creativecommons.org/licenses/by-nc/4.0/.

ORCID iDs

Edem Allado http://orcid.org/0000-0002-1760-6979

Mathias Poussel http://orcid.org/0000-0002-4386-8606

\section{REFERENCES}

1 Chi YM, Jung T-P, Cauwenberghs G. Dry-contact and noncontact biopotential electrodes: methodological review. IEEE Rev Biomed Eng 2010;3:106-19.

2 Lim YG, Kim KK, Park KS. Ecg measurement on a chair without conductive contact. IEEE Trans Biomed Eng 2006;53:956-9.

3 Ishijima M. Monitoring of electrocardiograms in bed without utilizing body surface electrodes. IEEE Trans Biomed Eng 1993;40:593-4.

4 Min SD, Kim JK, Shin HS, et al. Noncontact respiration rate measurement system using an ultrasonic proximity sensor. IEEE Sensors J 2010:10:1732-9.

5 Garbey M, Sun N, Merla A, et al. Contact-free measurement of cardiac pulse based on the analysis of thermal imagery. IEEE Trans Biomed Eng 2007;54:1418-26.

6 Humphreys K, Ward T, Markham C. Noncontact simultaneous dual wavelength photoplethysmography: a further step toward noncontact pulse oximetry. Rev Sci Instrum 2007;78:044304

7 Verkruysse W, Svaasand LO, Nelson JS. Remote plethysmographic imaging using ambient light. Opt Express 2008;16:21434.

8 Poh M-Z, McDuff DJ, Picard RW. Non-Contact, automated cardiac pulse measurements using video imaging and blind source separation. Opt Express 2010;18:10762.

9 Scully CG, Lee J, Meyer J, et al. Physiological parameter monitoring from optical recordings with a mobile phone. IEEE Trans Biomed Eng 2012;59:303-6

10 Lindberg LG, Tamura T, Oberg PA, PÅ Öberg. Photoplethysmography. Part 1. Comparison with laser Doppler flowmetry. Med Biol Eng Comput 1991;29:40-7.

11 Nakajima K, Tamura T, Miike H. Monitoring of heart and respiratory rates by photoplethysmography using a digital filtering technique. Med Eng Phys 1996;18:365-72.

12 Takano C, Ohta Y. Heart rate measurement based on a time-lapse image. Med Eng Phys 2007;29:853-7.

13 Moço A, Verkruysse W. Pulse oximetry based on photoplethysmography imaging with red and green light : Calibratability and challenges. J Clin Monit Comput 2021;35:123-33.

14 Parra JE, Da Costa G. Optical remote sensing of heartbeats. In: Allgood GO, Faust NL, eds. Proc. SPIE 4368, visualization of temporal and spatial data for civilian and defense applications. Orlando, FL, 2001: 113-21.

15 Fitzpatrick TB. The validity and practicality of sun-reactive skin types I through VI. Arch Dermatol 1988;124:869-71.

16 Liu H, Wang Y, Wang L. A review of non-contact, lowcost physiological information measurement based on photoplethysmographic imaging. Annu Int Conf IEEE Eng Med Biol Soc 2012;2012:pp.:2088-91.

17 Hollander JE, Carr BG. Virtually perfect? telemedicine for Covid-19. N Engl J Med 2020;382:1679-81.

18 Keesara S, Jonas A, Schulman K. Covid-19 and health care's digital revolution. N Engl J Med 2020;382:e82. 